\title{
A Case of Vasospastic Angina Resulting From Coronary Emboli in a Patient With Prosthetic Valves
}

\author{
Feridun Kosar, ${ }^{1}$ MD, Hakan Gullu, ${ }^{1}$ MD, Ibrahim SAHIN, ${ }^{2}$ MD, \\ Nusret ACIKGOZ, ${ }^{1} \mathrm{MD}$, Ergün TOPAL, ${ }^{1} \mathrm{MD}$, and Nevzat ERDIL, ${ }^{3} \mathrm{MD}$
}

\begin{abstract}
SUMMARY
In addition to coronary atherosclerotic disease, coronary thromboembolism can also lead to acute coronary syndromes. However, coronary thromboembolism due to prosthetic heart valves is very rare and not very well-known. It has Only a few cases have been reported. In this paper, we present a rare case with vasospastic angina pectoris secondary to coronary thromboembolism in a patient with prosthetic heart valves. (Jpn Heart J 2004; 45: 325-330)
\end{abstract}

Key words: Vasospastic angina, Variant angina, Coronary spasm, Prosthetic valve

CORONARY atherosclerotic disease is one of the most common causes of acute myocardial infarction. ${ }^{1)}$ In addition to atherosclerosis, coronary thromboembolism may also lead to acute coronary syndromes. ${ }^{2,3)}$ Several conditions such as dilated cardiomyopathy, chronic atrial fibrillation, infective endocarditis, intracardiac shunts, and hypercoagulable states are reported to cause coronary embolism, but there are only a few cases of coronary embolism in patients with a prosthetic heart valve reported in the literature. ${ }^{4-6)}$ We present a case of coronary saddle emboli causing variant angina pectoris in a patient with mitral and aortic valvular prostheses.

\section{CASE REPORT}

A forty-three year-old Turkish man with prosthetic heart valves in aortic and mitral positions was evaluated in our emergency department due to chest pain that had lasted for one hour. Detailed questioning on past medical history and physical examination revealed that he had no risk factors for coronary artery disease. However, he had undergone mitral and aortic valve replacement with mechanical prostheses for rheumatic valve disease 15 years earlier, and at that

\footnotetext{
From the ${ }^{1}$ Departments of Cardiology, ${ }^{2}$ Internal Medicine, and ${ }^{3}$ Cardiovascular Surgery, Faculty of Medicine, Inonu University, Turgut Ozal Medical Center, Malatya, Turkey.

Address for correspondence: Feridun Kosar, MD, Department of Cardiology, Faculty of Medicine, Inonu University, Turgut Ozal Medical Center, 44069 Malatya, Turkey.

Received for publication July 10, 2003.

Revised and accepted September 11, 2003. 
time, because of his young age coronary angiography was not performed. Oral anticoagulant therapy with warfarin had been initiated after the operation. However, without any reason, he discontinued warfarin therapy 15 days earlier to admission. His blood pressure was 120/70 $\mathrm{mmHg}$, and his heart rate was $85 \mathrm{bpm}$. On auscultation, heart sounds were arrhythmic and prosthetic valve clicks were normal. The ECG recording showed atrial fibrillation and ST- segment elevation in leads $\mathrm{V}_{1}-\mathrm{V}_{5}$ (Figure 1A). Heparin, aspirin, IV nitroglycerin, and beta-blocker

A
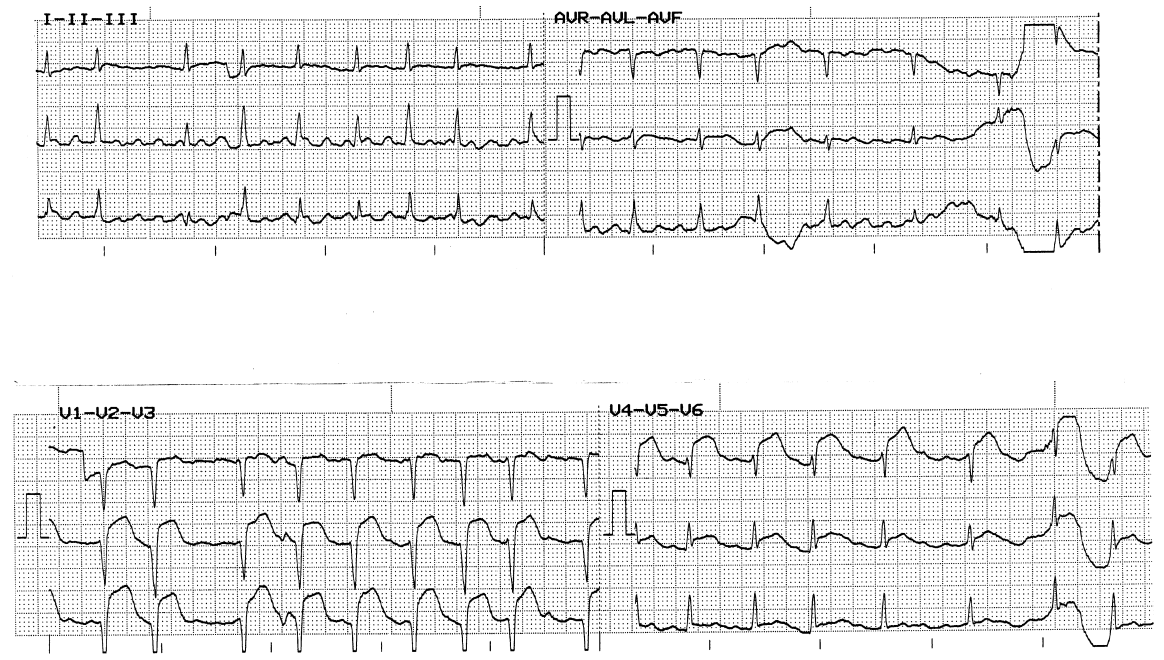

B
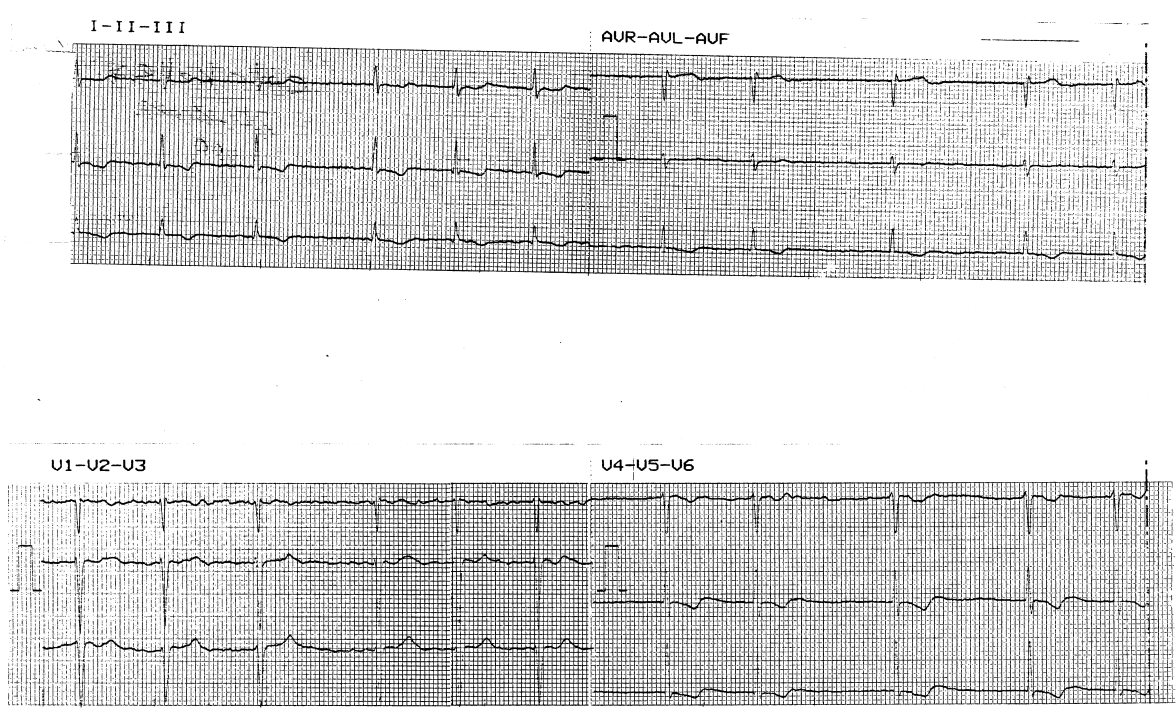

Figure 1. A: ECG recorded during an episode of chest angina showing atrial fibrillation and marked ST-segment elevation in leads $\mathrm{V}_{1}$ through $\mathrm{V}_{5}$. B: ECG recorded 10 minutes after episode; note that ST segment is almost back to normal. 
therapies were initiated. Ten minutes later his chest pain was relieved, and repeated ECG recordings demonstrated that the ST-segment elevations had normalized (Figure 1). Cardiac enzymes were in the normal ranges and INR (International Normalized Ratio) was in the subtherapeutic range (INR $=1.25)$. Based on these findings, he was diagnosed as having variant angina pectoris, and then admitted to the coronary care unit. The therapies initiated in the emergency department were continued, except that the beta-blocker therapy was changed to diltiazem therapy. Meanwhile, transthoracic echocardiography revealed left ventricular wall diskinesia, normal function of both mechanical prostheses, and no evidence of thrombi. On the seventh day of admission, coronary angiography was performed. Coronary angiography demonstrated that there was no sign of coronary atherosclerosis, including wall irregularity, but there was a saddle thrombus riding on the bifurcation point of the $\mathrm{LAD}$ and the first diagonal branch with TIMI 3 (Thrombolysis in Acute Myocardial Infarction) flow (Figures 2 and 3). Because of the saddle embolus causing no more than $50 \%$ stenosis in diameter, and having a high risk of distal embolization, no more coronary intervention was attempted to extract or dissolve the embolus. Also, the option of local thrombolysis was not considered since it was often ineffective and the patient had nonsignificant stenosis. Thus, the patient was given medical management with more aggressive anticoagulation to target an INR of 3.5. Since the patient was symptom-free and hemodynamically stable, he was discharged on diltiazem (180 mg daily), isosorbide mononitrate (40 mg daily), an oral anticoagulant, and aspirin

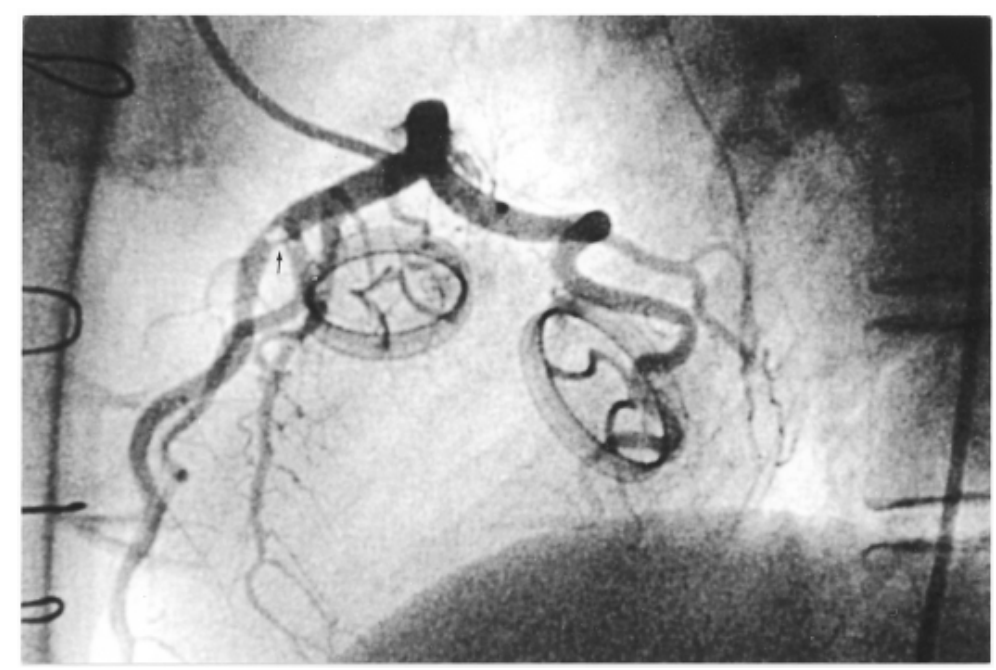

Figure 2. The arrow shows the appearance of saddle emboli from the left anterior oblique cranial projection. 


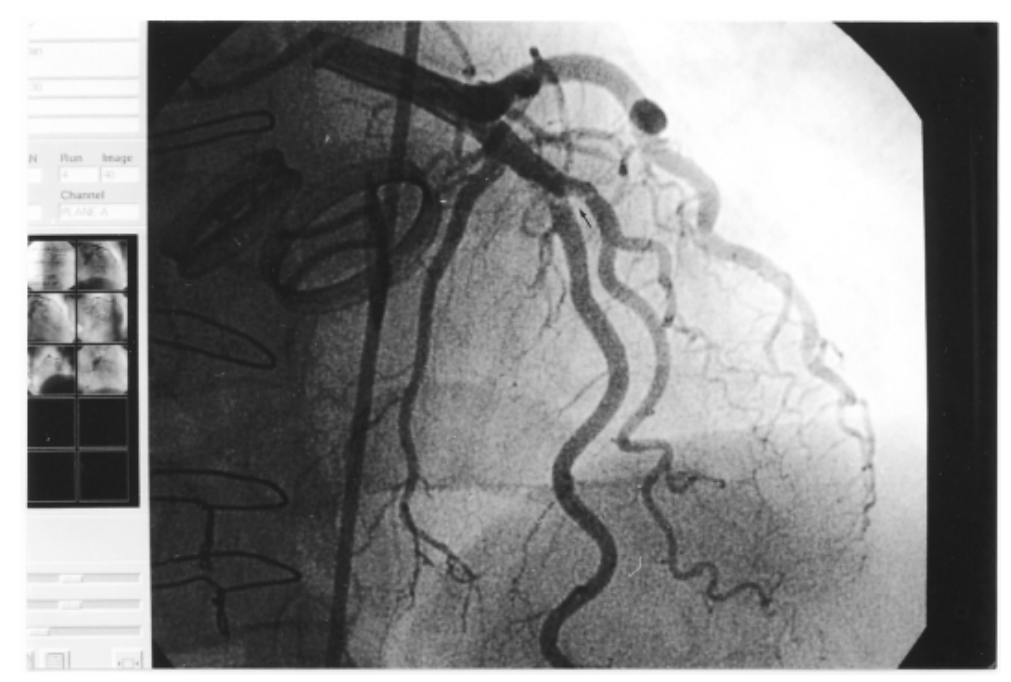

Figure 3. The arrow shows the appearance of saddle emboli from the right anterior oblique projection.

five days after achieving the target INR level on 2 consecutive days. The patient did not experience any further episodes of chest pain or complications during a 3month follow-up period. Therefore, we decided to continue with only medical treatment.

\section{DisCUSSION}

Four to seven percent of patients with acute myocardial infarction do not have underlying coronary artery disease. ${ }^{7)}$ Coronary embolism is included among nonatherosclerotic entities causing acute myocardial infarction and should be suspected in the presence of a valvular prosthesis, rheumatic valvular disease, chronic atrial fibrillation, dilated cardiomyopathy, infective endocarditis, and hypercoagulable states. ${ }^{8)}$ On the other hand, the diagnosis of coronary emboli is challenging and troublesome. Clinical suspicion is the main factor in making the diagnosis. In our patient, the risk factors which led to coronary emboli were atrial fibrillation, valvular disease, a mechanical prosthesis, and withdrawal of anticoagulant therapy. There is no doubt that any one of these risk factors could have caused the coronary emboli.

We have presented a patient with variant angina pectoris secondary to the embolus in his left anterior descending artery. The clinical presentation and ECG findings of our patient are relatively rare. During an episode of angina, marked $\mathrm{ST}$ elevations in leads $\mathrm{V}_{1}$ to $\mathrm{V}_{5}$ were recorded on the ECG. The ST-segment ele- 
vation was close to being normalized on a repeat ECG recorded 10 minutes after the symptoms terminated. The diagnosis of vasospastic angina was supported by the presence of chest pain associated with ST-segment elevations on the electrocardiogram, pain relief after administration of nitroglycerin, and no subsequent evidence of myocardial infarction. Coronary angiography was then performed to evaluate the coronary anatomy and determine the underlying pathology. Coronary angiograms showed partial occlusion of the LAD with TIMI 3 flow and a "horse-riding" thrombus on the bifurcation with the first diagonal branch, without evidence of an underlying atherosclerotic lesion. However, it is sometimes difficult to make a diagnosis and particularly to differentiate between coronary embolus and thrombus due to coronary artery disease based on only the angiographic appearance of the lesion. In this case, we thought that a thrombus arising from the left heart embolized to the left anterior descending artery via the left main coronary artery, and then the embolus was caught by the bifurcation point of the LAD at the first diagonal branch level. Also, this course of progression might have saved the patient from a myocardial infarction. Although the coronary events secondary to coronary embolism may be varied, the consequences depend on both the size of the embolus and the luminal diameter of the vessel where it is partially occluded. Because of his clinical features and the electrocardiography and coronary angiography findings, we strongly believe that the variant angina in our patient was caused by embolus-induced coronary spasm.

There is no specific therapy described for this rare condition. In general, anticoagulation, antiaggregation, and vasodilator therapy can initially be considered as the treatment of choice. However, today, there is no evidence-based therapy for coronary embolism, with the advance of PTCA and stenting the treatment of coronary embolism has remarkably changed into newer therapies. ${ }^{9}{ }^{10)}$ Several reports have described the intracoronary use of thrombolytics and intravenous administration of abciximab as an adjunct to PTCA and stenting in patients with coronary embolism. ${ }^{11,12)}$ In our patient, since embolus caused less than $50 \%$ diameter narrowing, and considering the fact that any coronary intervention can cause separation and/or mobilization of an embolus, we did not attempt any coronary intervention. Therefore, the patient was continued on the medical treatment.

Coronary embolism is an unusual clinical entity with an unknown prevalence, partly because of its difficult diagnosis. In patients with a prosthetic valve who are suffering from acute coronary syndromes with embolic risks factors, such as AF, valvular disease, a history of prior thromboembolic event, and mechanical prosthesis, the possibility of coronary embolism should be considered. Finally, if the clinical course is benign, interventional therapy for this condition is not mandatory. 


\section{REFERENCES}

1. Betriu A, Castaner A, Sany GA, et al. Angiographic findings 1 month after myocardial infarction: a prospective study of 259 survivors. Circulation 1982; 65: 1099-105.

2. Prizel KR, Hutchins GM, Bulkey BM. Coronary artery embolism and myocardial infarction, a clinicopathologic study of 55 patients. Ann Intern Med 1978; 88: 155-61.

3. Yutani C, Imakita M, Ueda-Ishibashi $\mathrm{H}$, et al. Coronary artery embolism with special reference to invasive procedures as the source. Mod Pathol 1992; 5: 224-9.

4. Kraus PA, Lipman J. Coronary embolus causing myocardial infarction. Intens Care Med 1990; 16: 215-6.

5. Shakil Aslam M, Sanghi V, Hersh S, Lakier JB. Coronary artery saddle embolus and myocardial infarction in a patient with prosthetic mitral valve. Cathet and Cardiovasc Interventions 2002; 57: 367-70.

6. Mouhcine Abid-Allah, Khalid AI Jubair. Acute right coronary embolus after aortic valve surgery. Asian Cardiovascular \& Thoracic Annals 2002; 10: 367-8.

7. Waller BF. Atherosclerotic and nonatherosclerotic coronary artery factors in acute myocardial infarction. Cardiovesc Clin 1989; 20: 29-104.

8. Charles RG. Coronary embolism in valvular heart disease. Q J Med 1982; 202: 147-61.

9. Mamby SA. Percutaneous transluminal coronary angioplasty of coronary embolism. Cathet Cardiovasc Diagn 1992; 25: 227-9.

10. Glazier JJ. Coronary embolism complicating aortic valve endocarditis: treatment with placement of an intracoronary stent. Clin Cardiol 1997; 20: 885-8.

11. Quinn EG. Coronary embolism following aortic and mitral valve replacement: successful management with abciximab and urokinase. Cathet Cardiovasc Diagn 1998; 43: 457-9.

12. Marti V, Romeo I, Garcia J, Guiteras P, Aminian N, Auge JM. Combined therapeutic strategy for multiple coronary thromboemboli. Cathet Cardiovasc Diagn 2000; 50: 343-5. 